SSC17-XIII-08

\title{
NORSAT-2: Enabling Advanced Maritime Communication with VDES
}

\author{
Laura M. Bradbury, Dumitru Diaconu, Simon Molgat Laurin, Clement Ma, Alex M. Beattie, and Robert E. Zee \\ Space Flight Laboratory, University of Toronto Institute for Aerospace Studies \\ 4925 Dufferin Street, Toronto, ON, Canada, M3H 5T6 \\ lbradbury@utias-sfl.net \\ Ivar S. Spydevold \\ Statsat AS \\ Drammensveien 165, 0212 Oslo, Norway \\ Hans Christian Haugli \\ Space Norway AS \\ Drammensveien 165, P.O. Box 66 Skøyen, 0212 Oslo, Norway \\ Jon Harr and Frank Udnæs \\ Norwegian Space Centre \\ Drammensveien 165, P.O. Box 113 Skøyen, 0212 Oslo, Norway
}

\begin{abstract}
Satellite AIS is well recognized as a system for vessel traffic monitoring and maritime safety. The growing demand for maritime data services has led to the development of a new VHF Data Exchange System (VDES), which will provide two-way communication at higher data rates than possible with current AIS systems. Within the VHF maritime frequency band (156.025-162.025 MHz), VDES integrates AIS with Application Specific Messages (ASM) channels, which have higher capacity and increased reliability. VDES will support the distribution of maritime data, including meteorological and hydrographic data and traffic information. Expanding VDES to a satellite platform will facilitate a global data exchange between ships and shore via satellite. NORSAT-2 is the first satellite to incorporate a VDES payload, in addition to an advanced AIS receiver, both of which are in-orbit reconfigurable software-defined radios developed by Kongsberg Seatex.

NORSAT-2 was developed and built by the Space Flight Laboratory (SFL) for Space Norway and the Norwegian Space Centre. With a mass of $16 \mathrm{~kg}$, the satellite design is based on SFL's Next-generation Earth Monitoring and Observation (NEMO) bus, which has proven flight heritage to be a robust and reliable microsatellite platform. To enable directional communication over VHF for the VDES payload, SFL has developed a three-element deployable Yagi-Uda antenna. The antenna is stowed during launch and deployed on-orbit upon command receipt. In addition to NORSAT-2's S-band telemetry and command system, the satellite also has an enhanced S-band feeder uplink which is capable of achieving data rates of up to 1 Mbps.
\end{abstract}

NORSAT-2 is scheduled to launch onboard a Soyuz in Q2 2017 into a $600 \mathrm{~km}$ polar sun synchronous orbit. It will be the fourth Norwegian satellite on-orbit and is highly anticipated to be one of the first satellites to supply VDES services, in addition to complementing Norway's existing satellite AIS network.

This paper describes the NORSAT-2 mission, with an emphasis on the unique and innovative aspects that are the VDES payload, deployable Yagi antenna, and enhanced S-band uplink.

\section{INTRODUCTION}

NORSAT-2 is a maritime-monitoring microsatellite built for the Norwegian Space Centre (NSC) by the Space Flight Laboratory (SFL) at the University of Toronto Institute for Aerospace Studies (UTIAS) in
Toronto, Canada. NORSAT-2 is a mission intended to carry out advanced ship tracking and VHF data exchange to continue Norway's expanding satellite constellation. 
NORSAT-2 leverages SFL's long history in building, launching, and operating low-cost small satellite missions. Since its establishment in 2008, SFL has successfully launched and operated 16 satellites including the first two Norwegian AIS satellites, AISSat-1 and AISSat-2. The first satellite in the constellation, AISSat-1, was launched on July 12, 2010 and is believed to be the first high performance nanosatellite to provide an observational service to government authorities. ${ }^{1}$

\section{MISSION OBJECTIVES}

NORSAT-2 is part of the Norwegian Space Centre's (NSC) initiative on small satellites for maritime surveillance. Norway reigns over seas with a total extension of more than 2 million square kilometres, which is more than six times larger than the mainland. In this body of water, Norway needs to manage natural resources, carry out search and rescue missions and safeguard sovereignty.

In cooperation with the Norwegian Coastal Administration (NCA) and the Norwegian Defence Research Establishment (FFI), NSC has therefore ensured the development of an operational system of AIS satellites capable of tracking vessels outside the coverage areas of land-based stations. As part of the Norwegian AIS satellite constellation, NORSAT-2 thus follows its predecessors, AISSat-1, AISSat-2 and NORSAT-1, whose main objectives are to assure continuous delivery of AIS data to Norwegian governmental users.

In addition to this main objective, NORSAT-2 carries an experimental payload for testing of a new VHF Data Exchange System (VDES), developed by Kongsberg Seatex for Space Norway. The project, which is supported by ESA and the International Association of Lighthouse Authorities (IALA), aims at demonstrating the feasibility of a VHF data exchange protocol via satellite to ships, which could eventually open for a new way of transmitting high priority information such as weather data, navigational routes, sea ice predictions, etc. Propagation measurements under an ESA contract are scheduled in July and August of 2017.

\section{VHF DATA EXCHANGE SYSTEM}

The maritime industry is digitizing most aspects of ship operation and the coastal administrations are developing a new data communications system called the VHF Data Exchange System (VDES). ITU released the first version of this standard ITU-R M.2092-0 in 2015. VDES supports terrestrial communications between ships and to stations near shore, whilst satellite communications is envisaged far from shore and particularly in the Arctic regions outside geostationary satellite coverage.

NORSAT-2 has an experimental two-way software defined VDES transceiver and will be used to develop propagation models and to demonstrate wide-area services such as Arctic ice chart distribution and notices to mariners. Two-way electronic navigation services (eNAV) are envisaged later when the standard is fully defined.

It is expected that future ship VDES transceivers will be combined with AIS into a single piece of equipment. This will have cost and installation benefits as hardware and antennas can, at times, be shared dynamically.

NORSAT-2 uses an $8 \mathrm{dBi}$ crossed Yagi antenna to minimize transmit power (and DC consumption) and to increase sensitivity. A typical mode for NORSAT-2 would be to point the antenna to a specific service area, such as NavArea XIX which is Norway's responsibility. The antenna is pointed by rotating the satellite dynamically during a pass.

VDES supports store-and-forward services, hence a LEO satellite would typically provide around 10 minutes of access time every 95 minutes around the Svalbard islands. Multiple satellites would reduce latency and provide redundancy.

\section{SATELLITE OVERVIEW}

The design of NORSAT-2 is based on SFL's flight proven NEMO (Next-Generation Earth Monitoring) bus design. The bus is $20 \times 27 \times 42 \mathrm{~cm}$ in size, with two pre-deployed $20 \times 50 \mathrm{~cm}$ solar wings. The entire spacecraft is $15.6 \mathrm{~kg}$ in mass. It was designed with a full complement of standard SFL electronics in addition to the payloads: AIS receiver, VDES payload, inspection camera, and S-band feeder uplink. The spacecraft exterior solid model is shown in Figure 1. 

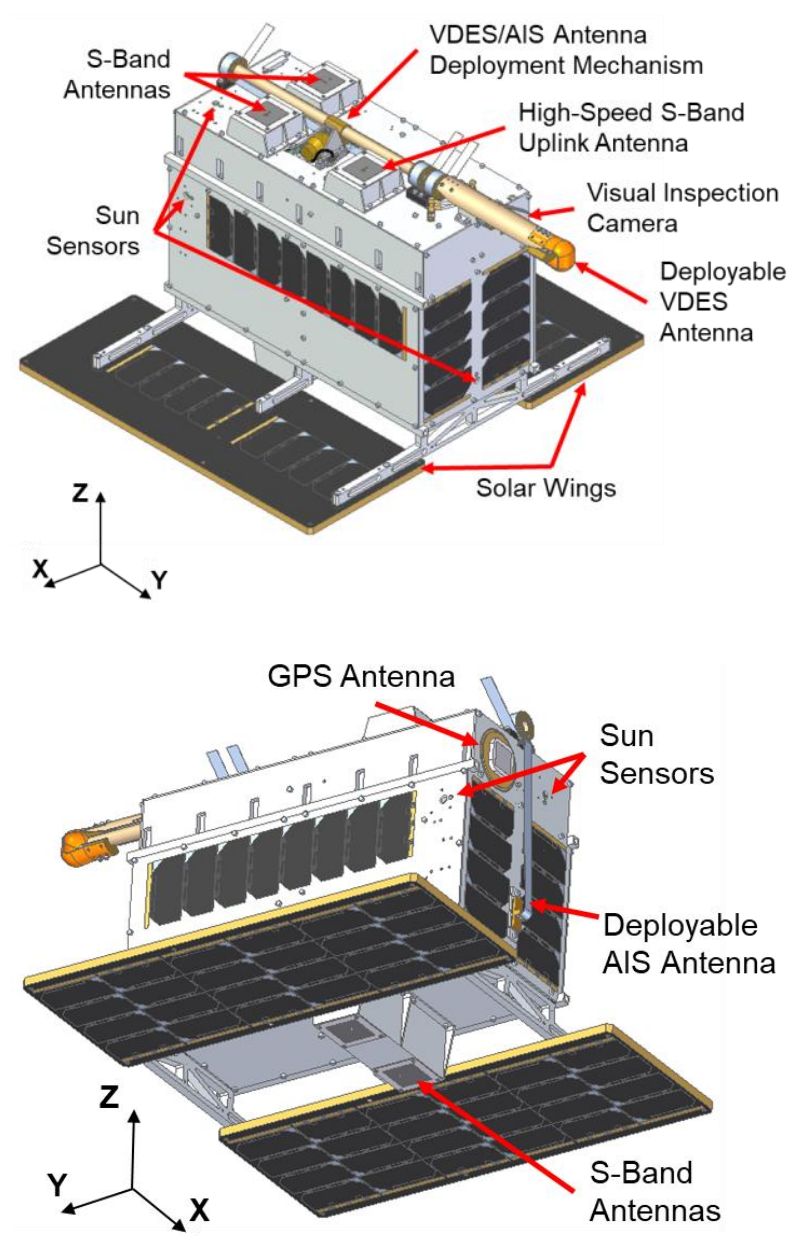

Figure 1: NORSAT-2 Exterior Solid Model

NORSAT-2 is intended to have several modes of operation, which are sun-pointing, limb-pointing within the orbit plane, and limb-pointing above a ground target. Limb pointing is done in order to optimize VDES and AIS operations, whereas sun-pointing is done in order to optimize power generation. One of the operational VDES modes is depicted in Figure 2.

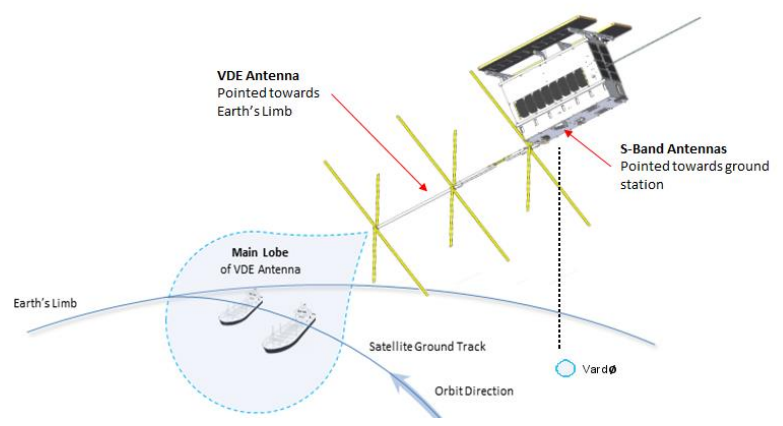

Figure 2: Limb-Pointing VDES Operations

\section{Mechanical Design}

The primary structure is built around a robust dual-tray concept which supports all subsystem components in a layout that facilitates rapid and flexible integration and testing. The satellite is fully enclosed by structural panels that provide support for attitude sensors and actuators, as well as solar cells, radio communications antennas, and other components. The structural subsystem is depicted in Figure 3.

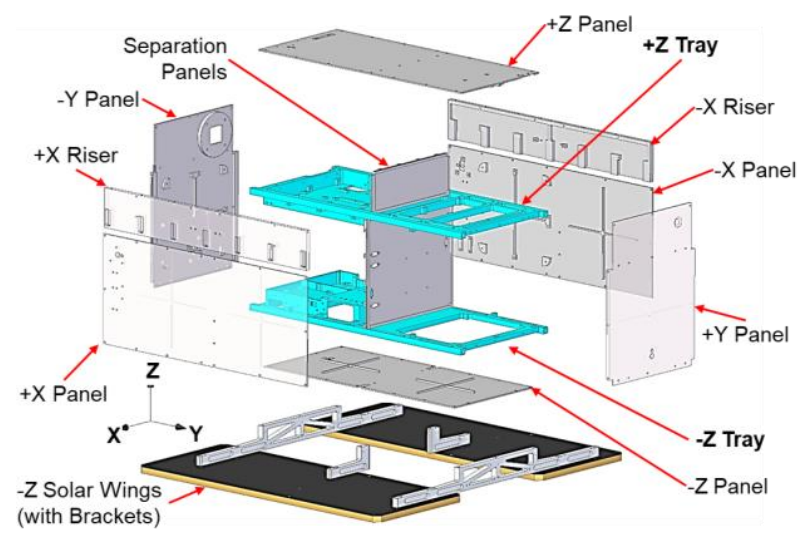

Figure 3: NORSAT-2 Structural Components

The two trays provide the primary load path between the satellite and its deployment system, SFL's XPOD Duo (eXoadaptable PyrOless Deployer). Figure 4 shows a rendition of NORSAT-2 in launch configuration inside the XPOD Duo. When compared to other deployers operating on the same principle, the unique design of the XPOD Duo reveals one of its chief advantages: it provides the microsatellite with two unconstrained faces to expand outside of the deployer's volume, making the NEMO platform highly adaptable to a large variety of missions and payloads. In the case of NORSAT-2, this flexibility enables two of the most noticeable features of this microsatellite, its two predeployed solar wings and its stowed VDES antenna. 


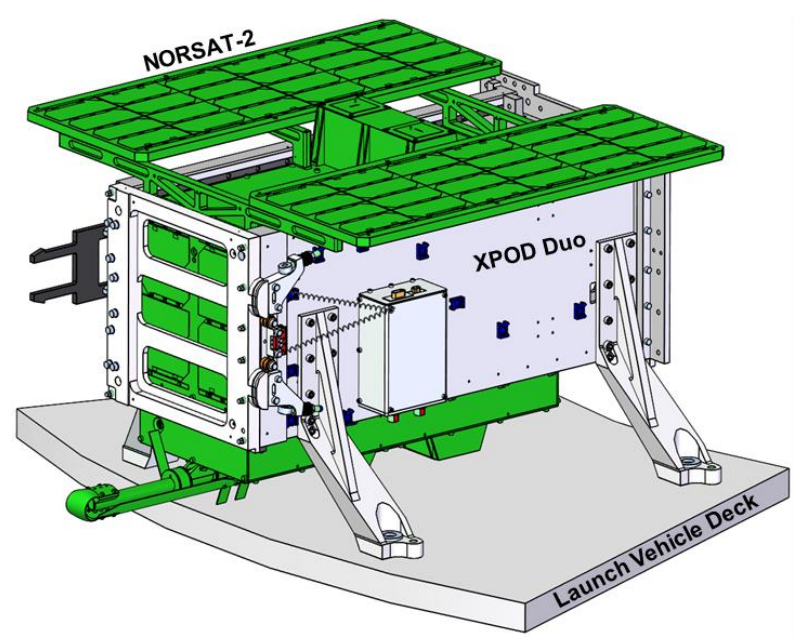

Figure 4: NORSAT-2 (green) Integrated in the XPOD Duo Deployer (grey)

In order to meet all structural integrity requirements while achieving a high payload mass fraction, the mechanical design of NORSAT-2 makes use of a wide range of high-performance materials. A mass breakdown of the system is presented in Table 1 .

Table 1: NORSAT-2 System Mass Budget

\begin{tabular}{|c|c|c|}
\hline Subsystem & Mass [kg] & Fraction \\
\hline Structural & 5.2 & $34 \%$ \\
\hline Thermal & 0.1 & $1 \%$ \\
\hline ADCS & 1.4 & $9 \%$ \\
\hline Power & 3.4 & $22 \%$ \\
\hline C\&DH & 0.1 & $1 \%$ \\
\hline Communications & 1.2 & $8 \%$ \\
\hline Payloads & 4.1 & $26 \%$ \\
\hline Total & $\mathbf{1 5 . 6}$ & $100 \%$ \\
\hline
\end{tabular}

\section{Command and Data Handling}

NORSAT-2 has three onboard computers, designated as a housekeeping computer (HKC), an attitude determination and control computer (ADCC), and a payload computer (POBC), each of which performs a distinct role. The $\mathrm{HKC}$ is responsible for basic housekeeping tasks such as regular collection of spacecraft telemetry, dispatch of commands, and managing radio communication. The ADCC is responsible for providing control over attitude hardware power switches and running attitude determination and control algorithms. The POBC is responsible for the control of the payloads, specifically the VDES, AIS and imager payloads, and interfacing with the S-band feeder uplink for bulk data and command reception.
Commanding can be done in real-time or via time-tag. Time-tagged commands allow for autonomous operations to occur outside of Earth station passes as they can be dispatched to a respective unit or software thread at a specified time. This functionality permits continuous AIS and VDES operations around the globe, even when real-time commanding is not possible.

\section{Electrical Power System}

The electrical power system supports the overall objective of NORSAT- 2 by providing a continuous and reliable power supply to the spacecraft using a series peak power tracking topology called the Modular Power System (MPS). The functionality of the MPS is divided into interchangeable cards located on a passive backplane. The system is capable of producing $48 \mathrm{~W}$ of generated solar power and can store approximately $108 \mathrm{Wh}$ using a single $3 \mathrm{~s} 2 \mathrm{p}$ Lithium ion battery. There is one body-mounted 8-cell solar string on each of the $\mathrm{X}$ and $\mathrm{Y}$ satellite faces, and two pre-deployed solar wings, containing a total of 64 solar cells (16 cells on the $+\mathrm{Z}$ side and 48 cells on the $-\mathrm{Z}$ side). The cells used are $\mathrm{GaInP} / \mathrm{GaAs} / \mathrm{Ge}$ triple junction solar cells with an efficiency of $30 \%$. Each cell is $40 \times 80 \mathrm{~cm}$ in size and is equipped with an integrated bypass diode.

When not executing operations with the VDES payload, the satellite remains in a maximum power generation attitude with the $-\mathrm{Z}$ satellite face sun pointing. This allows for continuous AIS operations and duty-cycled VDES operations in important areas of interest around Norway and around the globe.

\section{Telemetry and Command}

S-band is used for both uplink and downlink on NORSAT-2. In addition to the regular telemetry and command uplink and downlink system, NORSAT-2 also has an enhanced S-band feeder uplink.

The telemetry and command S-band uplink consists of a combiner, a band pass filter, a downconverter, and a UHF receiver. The S-band uplink forms the method with which commands are sent to the spacecraft for all mission functions, and therefore must be active at all times. Two patch antennas bonded on the $+\mathrm{Z}$ and $-\mathrm{Z}$ spacecraft faces are used to provide omnidirectional coverage.

The downlink is controlled by a custom-designed Sband transmitter from COTS components. Its data rate and modulation can be scaled on-the-fly from $32 \mathrm{kbps}$ to $2048 \mathrm{kbps}$. Scaling is automatically executed by ground software as the link with the Earth station improves and deteriorates throughout the pass. The antenna system used for the downlink on NORSAT-2 is 
an omnidirectional pair of patch antennas bonded to opposing satellite faces.

The high speed S-band feeder uplink is another customdesigned board capable of achieving uplink speeds of up to $1 \mathrm{Mbps}$. The purpose of including the separate feeder uplink is to support VDES operations. It is intended to allow the upload of large waveform files to the VDES payload. The S-band feeder uplink has a single patch antenna, bonded to the $+\mathrm{Z}$ face of the satellite.

\section{Attitude Determination and Control Subsystem}

The attitude determination sensors are comprised of a three-axis magnetometer, six fine sun sensors, and a three-axis rate sensor, which are all designed and built at SFL. Actuation is provided by three orthogonal reaction wheels and three orthogonal vacuum-core magnetorquers. The reaction wheels are used for fine pointing control and the magnetorquers are used mainly for reaction wheel desaturation. A GPS receiver is also present onboard for orbital position knowledge and precise timing.

The Attitude Determination and Control subsystem (ADCS) can operate in a number of control modes: Safe, Passive, B-dot, Wheel Momentum Control, and Three-Axis Control. Three-Axis Control is the primary operational attitude throughout the course of the mission and can be used in several useful sub-modes, including the previously mentioned limb pointing and ground target tracking. Ground target tracking is used for passes over the Earth station. In this mode, the spacecraft tracks a static target on the ground with a specified body axis while a second body axis is constrained to a second desired axis.

Momentum management is performed in parallel with active control tasks and can be enabled or disabled by an operator as needed. The desired spacecraft inertial angular momentum is entered as a setpoint, and the magnetorquers are actuated to regulate the wheel speeds while simultaneously holding the desired attitude.

\section{AIS Payload}

One of the payloads onboard NORSAT-2 is an advanced Kongsberg Seatex AIS receiver, which operates across the maritime frequency band from $156 \mathrm{MHz}$ to $163 \mathrm{MHz}$. The AIS antenna is a deployable tape-measure VHF monopole design that is held down along with the Yagi-Uda antenna during launch.

\section{Deployable VDES Antenna Overview}

The VDES antenna is a high gain, directional, folded dipole Yagi-Uda antenna with three elements in crossed configuration. It operates in the VHF maritime band, and its phase quadrature feed scheme allows it to produce circular polarization. At $62 \times 62 \times 73 \mathrm{~cm}$ overall dimensions, its deployed geometry exceeds the total size of the NORSAT-2 satellite body. Figure 5 shows the NORSAT-2 configuration with the VDES and AIS antennas deployed.

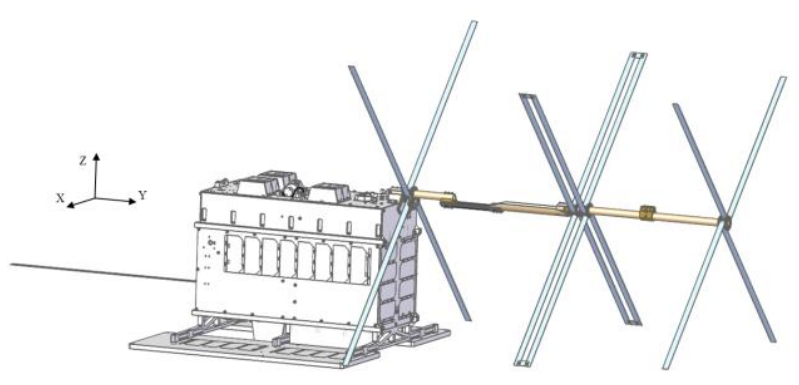

Figure 5: NORSAT-2 Deployed Configuration

The deployable concept was adopted early in the design cycle, after a trade study that included a pre-deployed version of the same antenna as well as variations of helical and loop Yagi antennas.

The ability to stow the antenna for launch enables a substantially lighter design that can nonetheless meet structural strength and stiffness requirements. In addition, the launch volume of the satellite is minimized, increasing compatibility with several launch vehicles and mounting configurations. The stowed VDES antenna configuration (Figure 6 and Figure 7) integrates a hold-down mechanism for NORSAT-2's monopole AIS antenna as well.

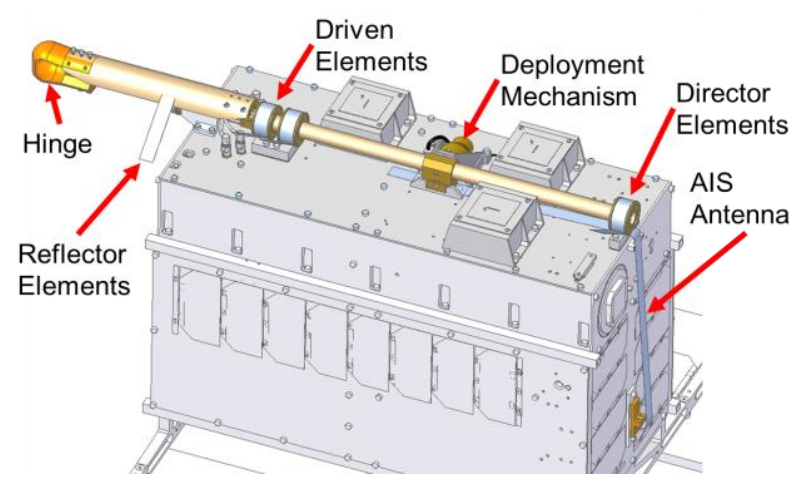

Figure 6: NORSAT-2 Stowed Antenna Configuration 


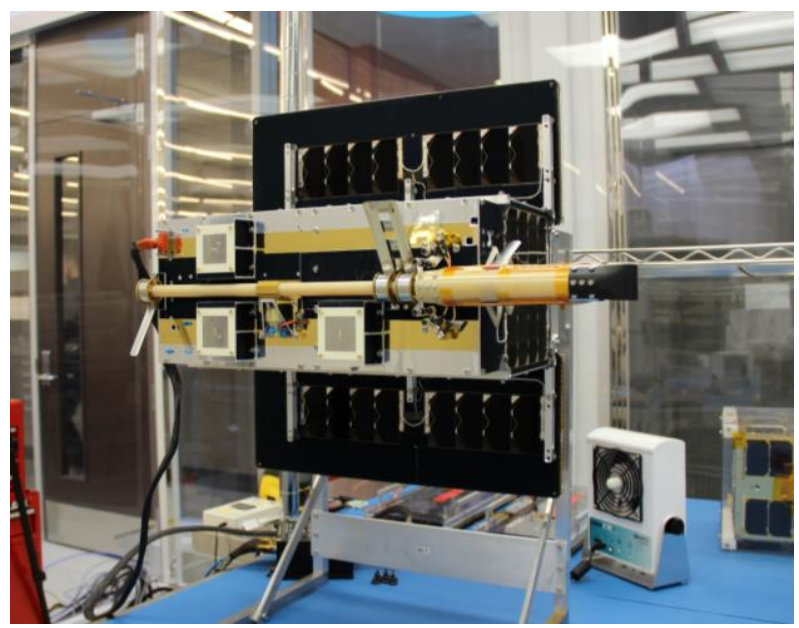

Figure 7: Stowed VDES Antenna aboard NORSAT-2 (Flight)

\section{VDES ANTENNA MECHANICAL DESIGN VALIDATION AND PERFORMANCE VERIFICATION}

The flight configuration of the VDES antenna was validated through a thorough test campaign involving radio-frequency (RF) performance testing, deployment testing, and environmental testing. Each test was conducted according to SFL standards and the results verified with regards to both internal and customer requirements. The successful completion of the test campaign ensures that the VDES antenna will perform as desired in orbit with a high degree of confidence.

\section{Pattern and EIRP Testing}

Pattern testing of the VDES antenna was conducted with the goal of verifying the gain and polarization performance of the deployable design in relation to simulation results and customer specifications. The test was conducted coarsely in an early design phase to verify the prototype's performance, and with finer precision on the flight model; the results demonstrated a performance that met or exceeded nominal gain requirements at both the receive and transmit frequencies for all spacecraft orientations. An example of the pattern results is presented in Figure 8 below.

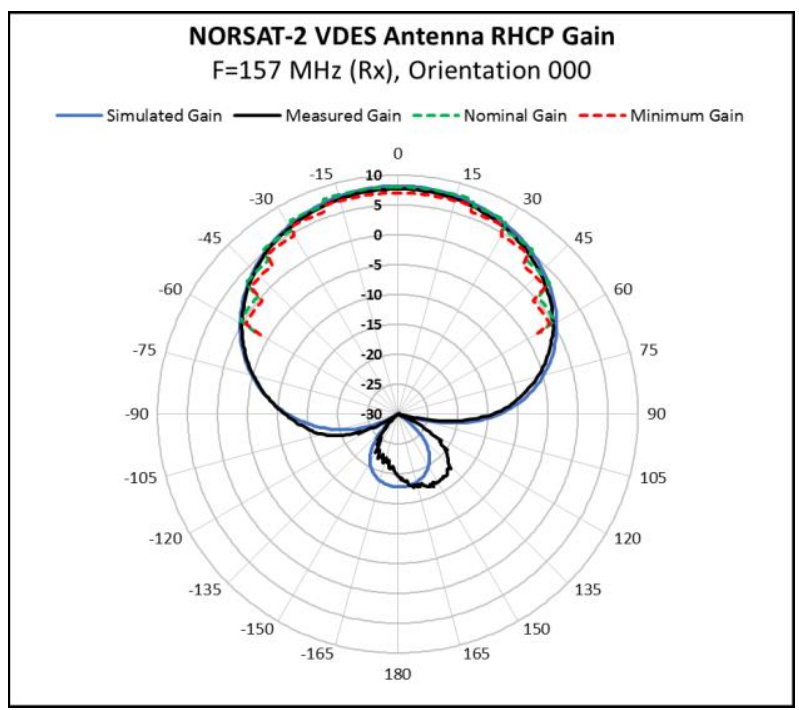

Figure 8: VDES Antenna Pattern - Receive Frequency, Nominal Orientation

Additionally, the maximum variation in the Equivalent Isotropic Radiated Power (EIRP) of the payload was measured for a nominal boresight orientation between the VDES antenna and a ground receiver, as well as at orientations with offsets in elevation and azimuth of up to $30^{\circ}$. The results confirmed the proper functioning of the VDES antenna transmission mode.

\section{Qualification Vibration Testing}

Due to the considerable differences between the VDES antenna and previous deployable structures designed by SFL, the antenna was subjected to a full unit-level qualification vibration test, including sinusoidal vibration tests, random vibration tests and shock tests in all three axes. Following these tests, it was observed that the pre-load applied to each of the antenna coils had been maintained, and the antenna's structural integrity was verified. Additionally, following integration with the flight spacecraft, the antenna was subjected to acceptance-level loads during the spacecraft acceptance vibration campaign.

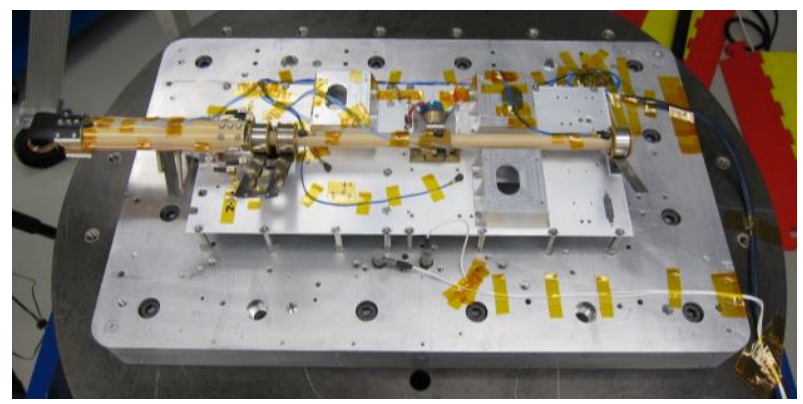

Figure 9: VDES Antenna Unit-Level Qualification Vibration Test 


\section{Qualification Thermal Vacuum Testing}

Further environmental testing was performed on the flight VDES design in SFL's Thermal Vacuum (TVAC) chamber. Full unit-level qualification thermal cycling was conducted on two antennas simultaneously, with one unit being deployed at the hot operational temperature $\left(+60^{\circ} \mathrm{C}\right)$, and the other at cold $\left(-20^{\circ} \mathrm{C}\right)$. The test confirmed the deployment mechanism's functionality at both temperature extremes, as well as the antenna's structural integrity and stable electrical connectivity in both a stowed and deployed state across the expected operational temperatures.

\section{Deployment Testing}

Deployment testing was conducted at regular intervals throughout the antenna design phases in order to verify that the antenna would reliably release, unfold, and stabilize to its fully deployed state. These tests were conducted prior to and following any design adjustments or qualification tests in order to reveal any changes in either the deployment dynamics or the functionality of the deployment mechanism.

Initial testing was conducted in atmosphere, while further deployment testing on the flight configuration was conducted in vacuum. These later tests exposed and helped characterize effects resulting from absence of air friction compared to the first stages of atmosphere testing: faster deployment of the individual coiled elements and generally more energetic motions, along with slower overall stabilization from the stowed state to the stationary, fully deployed state. Generally, the deployment consists of two phases, one approximately two seconds in duration in which a rapid and ample transition to deployed shape takes place, followed by the second phase of approximately 30 seconds duration of small oscillation damping. Figure 10 displays the deployed VDES antenna following a test in SFL's TVAC chamber.

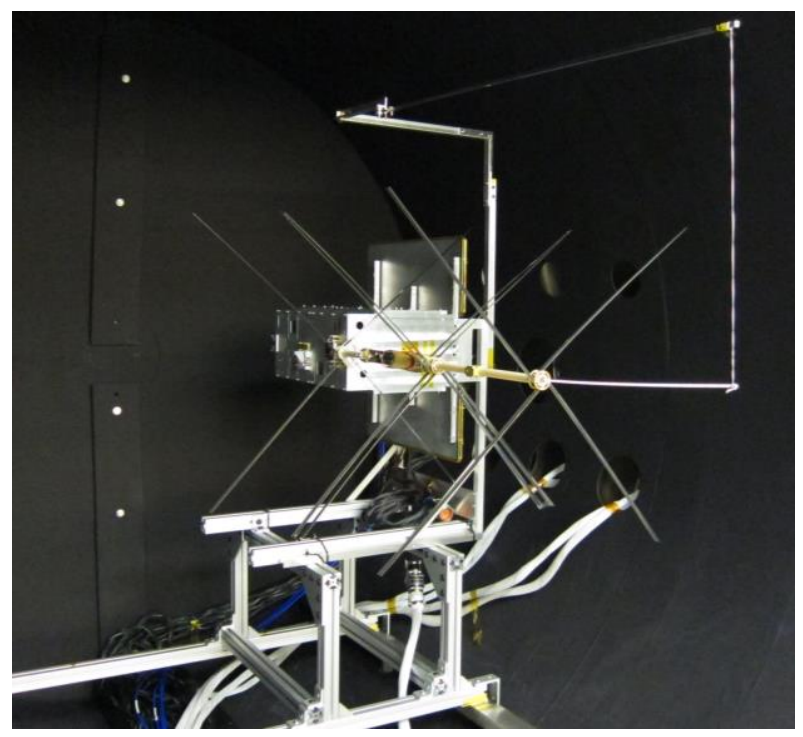

Figure 10: VDES Antenna Deployment Testing in TVAC

Additionally, multiple consecutive deployments were conducted to verify consistency in the antenna's deployment behavior as well as its final stabilized state. Prior to flight, a total of over 100 deployments were conducted with the antenna prototype, qualification model and flight model over the course of the antenna's development.

\section{LAUNCH AND EARLY OPERATIONS}

NORSAT-2 will be launched on a Soyuz from the Baikonur Cosmodrome in Kazakhstan. In addition to the 71 other satellites on the launch vehicle, it will also launch alongside NORSAT-1, which is another Norwegian satellite with an AIS instrument and two other science payloads.

Two Earth stations will be used for commissioning, one located in Vardø, Norway and the other located at Svalbard. Antenna control is performed by Kongsberg Satellite Services (KSAT). The operations centre for NORSAT-2 is located at Statsat AS in Oslo, Norway.

\section{CONCLUSIONS}

NORSAT-2 is highly anticipated to be the first satellite on-orbit to demonstrate VDES capability. The NORSAT-2 mission will demonstrate Norway's capability of providing data important for maritime surveillance and commerce, and SFL's ability to provide satellite technology capable of supporting these payloads.

\section{ACKNOWLEDGMENTS}

The authors would like to gratefully acknowledge the staff and students at SFL for their support and 
contributions throughout the duration of this program. The authors would also like to thank those who worked on the NORSAT-2 program from Statsat, Space Norway, and the Norwegian Space Centre.

\section{REFERENCES}

1. Narheim, B.T. et al., "AISSat-1 Early Results," Proceedings of the $25^{\text {th }}$ Annual AIAA/USU Conference on Small Satellites, Logan, UT, August 2011. 